\title{
All that shakes is not epilepsy
}

\author{
YM Hart \\ Consultant Neurologist, Royal Victoria Infirmary, Newcastle upon Tyne, UK
}

This review is based in part on Dr Hart's lecture at the RCPE Symposium on Neurology in Edinburgh on 16 November 2011.

\begin{abstract}
Experience from the clinic suggests that many people equate the term 'epilepsy' with the occurrence of convulsions, with the corollary that attacks involving shaking are likely to be due to epilepsy. However, just as many seizure types do not involve shaking, the differential diagnosis of intermittent shaking is wide and includes vasovagal syncope, cardiac disorders, concussive convulsions, psychogenic non-epileptic seizures, 'shaking transient ischaemic attacks', parasomnias, breath-holding attacks in children, hypoglycaemia and movement disorders.
\end{abstract}

\author{
Correspondence to YM Hart \\ Department of Neurology, \\ Royal Victoria Infirmary, \\ Newcastle upon Tyne \\ NEI 4LP, UK
}

tel. $+44(0) 1912823227$

e-mail yvonne.hart@nuth.nhs.uk

KEYWORDS Epilepsy, seizure, convulsion, syncope, tonic clonic

DECLARATION OF INTERESTS No conflicts of interest declared.

\section{SYNCOPE}

Syncope is the condition which is most commonly misdiagnosed as due to seizure activity. Although the layperson's view of fainting is that it involves sudden loss of consciousness, usually preceded by a warning of lightheadedness, accompanied by pallor, loss of body tone and quick recovery, the reality is that other symptoms and signs are commonly associated. The features of syncope were clearly delineated in a study by Lempert et al.,' in which 56 medical students fainting as a result of carrying out the 'mess lark' were recorded on video. Sixty per cent reported auditory or visual hallucinations. Forty-six students lost consciousness completely and fell and $90 \%$ of these showed myoclonic jerking, usually multifocal arrhythmic jerks. Other movements such as head turning, oral automatisms and righting movements occurred in $70 \%$, and initial upward deviation of the eyes was also common. The duration of the episodes was brief (12.1 +/- 4.4 seconds). This study suggests that features commonly used to delineate seizures, such as jerking or automatisms, are poor at differentiating seizures from syncope. Important factors which should be actively sought in making the diagnosis of vasovagal syncope are the presence of a precipitating factor such as pain or strong emotion, the occurrence of a typical aura (common presyncopal symptoms include a feeling of light-headedness, greying of vision, muffled hearing and nausea), brief duration and the lack of significant postictal confusion, although patients may feel 'drained' afterwards. The duration of loss of consciousness may be prolonged if the patient is supported in the upright position during a syncopal episode, when they may go on to develop more florid convulsive movements due to cerebral hypoxia. A more prolonged loss of consciousness may also occur if a head injury causing a concussive convulsion results from syncope (see below). Both concussive convulsions and concussive syncope are distinguished from unprovoked seizures by the UK Driver and Vehicle Licensing Agency and do not usually result in loss of driving privileges.

\section{CARDIAC DISORDERS}

Cardiac disorders such as long QT syndrome or Brugada syndrome, which can lead to ventricular arrhythmias, can present as syncope or seizures and every patient presenting with seizure-like episodes or transient loss of consciousness should undergo an electrocardiogram (ECG), since these conditions can result in sudden death. Unlike vasovagal syncope, there may not be any warning. If doubt about the diagnosis remains, other cardiac investigations such as echocardiogram, 24-hour Holter monitoring, or monitoring using an implantable loop recorder may be helpful. In those patients in whom a cardiac pacemaker is considered appropriate, consideration should be given to carrying out a magnetic resonance imaging (MRI) scan beforehand if there is any ongoing doubt about the possibility of seizures.

\section{CONCUSSIVE CONVULSIONS}

Concussive convulsions were described by McCrory et al. $^{2}$ in a study of convulsions occurring at the time of acute head injury sustained during Australian rules football or rugby league football. The authors identified 22 such cases, which were characterised by convulsions developing within two seconds of impact. Typically a brief tonic phase was followed by bilateral myoclonic jerking 
which was frequently asymmetrical. Such movements lasted up to 150 seconds, following which the players awoke with neuropsychological and behavioural features similar to mild concussion. The players were followed up for 1-13 years: all were able to return to sport within two weeks, and none went on to develop epilepsy. No permanent or structural injury developed.

In practical terms a common scenario is that people sustain a head injury in the course of a fall, and in these circumstances it is usually difficult to know whether the convulsion is the cause or effect of the fall.

\section{PSYCHOGENIC NON-EPILEPTIC SEIZURES}

Psychogenic non-epileptic seizures (PNES) are probably the second most common condition after syncope to be misdiagnosed as epileptic seizures. They may take several forms. ${ }^{3}$ Most commonly they involve motor phenomena, often with thrashing or flailing of the limbs, broadly resembling tonic-clonic seizures. Frequently reported features in PNES include pelvic thrusting, opisthotonus, rocking, kicking and cycling movements. All such features (as well as a rapid recovery, also common after PNES) can also occur in people with frontal lobe seizures, which are most at risk of being misdiagnosed as PNES, but frontal lobe seizures are usually shorter, often lasting less than one minute, in contrast to PNES which are often prolonged. Turning to a prone position is more common in patients with frontal lobe seizures. Some reports suggest that PNES are uncommonly reported during sleep: however, in a study by Duncan et al., ${ }^{4} 59 \%$ of people with PNES gave a history of such episodes. Also contrary to popular belief, self-injury may occur in association with PNES, being reported by $40 \%$ of patients in one study: ${ }^{5}$ the same study reported tonguebiting in $44 \%$ of patients with PNES. However, burns occurring during a seizure confer a high likelihood that the seizure is epileptic. Urinary incontinence may occur in either epileptic or non-epileptic seizures. Other features which may point towards the diagnosis are eyeclosure and absence of postictal symptoms, both of which are more common in PNES. ${ }^{3}$ Hyperventilation may occur in people with PNES but cyanosis should steer physicians towards a diagnosis of epilepsy.

Not all patients with PNES exhibit active motor phenomena: in some, seizures are manifest by a 'swoon', in which the patient is unresponsive and often atonic, without motor symptoms: relatives may describe them looking as if they are asleep. Prolonged staring can also occur. ${ }^{3}$ Not all PNES occur at times of acute stress (and stress may also be a trigger for epileptic seizures). Syed et al. ${ }^{6}$ found that preserved awareness, eye flutter and the ability of bystanders to intensify or alleviate seizures were indicative of PNES, while abrupt onset, eyeopening/widening and post-ictal confusion or sleep suggested epileptic seizures. Other features which have been suggested as distinguishing features are that side-to -side head movements are more common in PNES, while frontal lobe seizures are usually stereotyped, which is not always the case with PNES..$^{78}$

Antiepileptic drugs (AED) and trials of treatment should be avoided: it is very difficult to discontinue AEDs once started and there may be a placebo effect even in patients with non-epileptic seizures. The fact that they have been used also suggests to future carers that the diagnosis of epilepsy was secure. Many people with PNES have frequent seizures such that electroencephalography (EEG)-videotelemetry is feasible. However, the absence of epileptic changes on EEG in some patients with frontal seizures and those with minor simple partial seizures should be noted. There is also a proportion of people with epilepsy who go on to develop PNES. Care should therefore be exercised in withdrawing medication from people later found to have PNES, particularly if the original diagnosis of epilepsy was made elsewhere, unless clear criteria for the original diagnosis are available.

\section{SLEEP DISORDERS}

Seizures that occur in sleep (particularly frontal lobe seizures, which typically occur in early sleep) may be confused with parasomnias, including periodic limb movements of sleep, rapid eye movement (REM) sleep behaviour disorder, night terrors in children and sleep walking. Sleep walking and talking occur commonly in children, but are less frequently seen in adults. Night terrors typically affect young children: they occur in slow wave sleep and the child appears agitated, frightened and may cry or scream. The night terrors usually last a number of minutes, sometimes as long as an hour, but are not remembered by the child. They rarely persist into adolescence.

\section{PERIODIC LIMB MOVEMENTS OF SLEEP}

Periodic limb movements of sleep typically occur in nonREM sleep and involve extension of the big toe with flexion at the ankle, knee and sometimes hip, though arm movements may also occur. The movements usually last 5-10 seconds and recur at intervals of 20-40 seconds for periods of minutes to hours at a time. Periodic limb movements of sleep become increasingly common in older age, occurring in 50\% of people over the age of 65 years. Restless legs (in which people typically experience an unpleasant sensory disturbance in the legs, usually in the evening, associated with the urge to move the legs to ease these sensations) may occur together with periodic limb movements of sleep. 


\section{REM SLEEP BEHAVIOUR DISORDER}

Like periodic limb movements of sleep, REM sleep behaviour disorder is also more common in older age, typically occurring in people over the age of 50 years: the overall prevalence is thought to be approximately $0.5 \%$. About $80 \%$ of cases occur in men and in up to about $50 \%$ of cases, this condition occurs in association with, or precedes, a neurodegenerative disorder, particularly Parkinson's disease, Lewy body dementia and multiple system atrophy. A significant proportion of people present with injuries sustained in sleep, including fractures. REM sleep behaviour disorder is characterised by loss of the voluntary muscle atonia which normally occurs with REM sleep, resulting in the acting out of dreams which may be violent. The dreams often appear to be extremely vivid and bed partners are at significant risk of injury. If the patient is awoken in an attack, he or she is often able to remember the dream resulting in the witnessed activity.

Because it occurs in REM sleep, this disorder usually occurs later in the sleep cycle, whereas frontal lobe seizures often occur as the patient is drifting off to sleep (though they may also occur on awakening). Whereas frontal lobe seizures are often stereotyped, the features of different episodes of REM sleep disorder may vary. It is also unusual for directed violence to occur in seizures, whereas it is relatively common in REM sleep disorder, where the patient's partner is often mistaken for the aggressor in a dream. Fortunately, clonazepam is an effective treatment for the vast majority of people with REM sleep behaviour disorder.

\section{SHAKING TIAS}

Usually the differentiation of transient ischaemic attacks (TIA) from focal seizures is not difficult. Whereas seizures commonly involve positive phenomena such as motor activity or paraesthesiae, the symptoms of TIAs are more often negative, for example weakness or numbness (the distinction is not absolute, however, and negative symptoms such as loss of vision, or weakness, can also occur as ictal symptoms). A further distinction is that some spread of symptoms is usually discernible in seizures, whereas TIAs are generally of sudden onset (again, there may be difficulty with some forms of seizure, for example where dysphasia is the main symptom). However, TIAs can occasionally present with episodes of shaking, so-called 'shaking TIAs'. Baquis et al.' described eight patients with carotid stenosis who presented with brief, involuntary, coarse, irregular wavering movements or trembling involving either the upper limb alone, or the arm and leg on the same side. Six underwent vascular surgery, five with benefit, while one subsequently had a stroke affecting the same vascular territory. The other two were treated with antiplatelet agents with benefit. Although in one patient the events were precipitated by standing, in others they occurred while the patient was in the supine or sitting position and the possibility of carotid occlusive disease should be considered in patients with similar presentations.

\section{FACIOBRACHIAL DYSTONIC SEIZURES}

Faciobrachial dystonic seizures should also be included in the differential diagnosis of shaking attacks. There is ongoing debate about their actual nature (i.e. whether or not they are due to seizure activity) and even if they do represent a form of seizure, they are often resistant to standard antiepileptic drugs (only 18\% responding), improving instead with immunotherapy. They were described by Irani et al $^{10}$ and further characterised by Irani et al in 201 I." The initial description was of three 69-year-old patients who developed the subacute onset of facial grimacing and dystonic posturing of one or both arms, the events being stereotyped, lasting seconds only, and occurring very frequently (up to 70 times per day). They were not clearly associated with loss of consciousness (though the later paper does describe brief loss of awareness in some attacks in $66 \%$ of patients). The patients described also experienced occasional tonic-clonic seizures. All were found to have anti-voltage gated potassium channel antibodies: their seizures were resistant to treatment with standard antiepileptic drugs, but responded to immunotherapy. In their later study the authors described 20 patients aged from 36 to 83 years with similar symptoms. There was a male:female ratio of approximately $2: I$. In the majority of patients anti-leucine-rich anti-glioma I protein (anti-Lgil) antibodies were present. The faciobrachial dystonic seizures were frequently found to herald the development of limbic encephalitis.

\section{OTHER 'SHAKING' DISORDERS}

While not usually causing a problem diagnostically, movement disorders, including Parkinson's disease, tics and others may sometimes be mistaken for seizures. Breath-holding attacks, which may occur in children who are thwarted or have hurt themselves, may also sometimes be associated with brief jerking.

\section{SUMMARY}

It is clear that a wide range of paroxysmal neurological events can be associated with shaking or jerking movements and may be mistaken for epileptic seizures. A careful history and eyewitness description is crucial to their differentiation from seizures. Particularly important aspects of the case history include the circumstances of the attack and any trigger factors, clinical features, duration, length and nature of the post-ictal period and any recurrence. Although investigation is important and 
may be helpful in diagnosis, routine EEG has only a limited role to play, since only $35 \%$ of people with epilepsy consistently show epileptiform abnormalities interictally (and 15\% never show such activity interictally). ${ }^{2}$ Syncope and psychogenic non-epileptic seizures are commonly seen in epilepsy or first seizure clinics, while sleep disorders, vascular conditions and movement disorders also need to be considered in the differential diagnosis.

\section{REFERENCES}

I Lempert T, Bauer M, Schmidt D. Syncope: a videometric analysis of 56 episodes of transient cerebral hypoxia. Ann Neurol 1994; 36:233-7. http://dx.doi.org/10.1002/ana.410360217

2 McCrory PR, Bladin PF, Berkovic SF. Retrospective study of concussive convulsions in elite Australian rules and rugby league footballers: phenomenology, aetiology and outcome. BMJ 1997; 3|4:|7|-4. http://dx.doi.org//0.1 |36/bmj.3 |4.7075. I7 |

3 Mostacci B, Bisulli F, Alvisi L et al. Ictal characteristics of psychogenic non-epileptic seizures: what we have learned from video/EEG recordings: a literature review. Epilepsy Behav 201 I; 22:144-53. http://dx.doi.org/10.1016/j.yebeh.2011.07.003

4 Duncan R, Oto M, Russell AJ et al. Pseudosleep events in patients with psychogenic non-epileptic seizures: prevalence and associations.J Neurol Neurosurg Psychiatry 2004; 75: I009-12. http:// dx.doi.org/ / 0. I I36/jnnp.2003.022632

5 Peguero E, Abou-Khalil B, Fakoury T et al. Self-injury and incontinence in psychogenic seizures. Epilepsia 1995; 36:586-91. http://dx.doi.org/I0.I I I I/j.I528-I I57.1995.tb02572.x

6 Syed TU, LaFrance WC, Kahriman ES et al. Can semiology predict psychogenic nonepileptic seizures? A prospective study. Ann Neurol 20 I I 69:997-1004. http://dx.doi.org/I0.1002/ana.22345

7 Lesser RP. Psychogenic seizures. Neurology 1996; 46:1499-507.

8 Saygi S, Katz A, Marks DA et al. Frontal lobe partial seizures and psychogenic seizures:comparison of clinical and ictal characteristics. Neurology 1992; 42: 1274-7.

9 Baquis GD, Pessin MS, Scott RM. Limb shaking - a carotid TIA. Stroke 1985; 16:444-8. http://dx.doi.org/I0.II6I/0I.STR.16.3.444

10 Irani SR, Buckley C, Vincent A et al. Immunotherapy-responsive seizure-like episodes with potassium channel antibodies. Neurology 2008; 7I:I647-8. http://dx.doi.org/I0.I2 I2/0I.wnl.0000326572.93762.5 I

I I Irani SR, Michell AW, Lang B et al. Faciobrachial dystonic seizures precede Lgil antibody limbic encephalitis. Ann Neurol 201I; 69:892-900. http://dx.doi.org//0.1002/ana.22307

12 Marsan CA, Zivin LS. Factors related to the occurrence of typical paroxysmal abnormalities in the EEG records of epileptic patients. Epilepsia 1970; I I:36 I-8I.http://dx.doi.org/I0. I I I I/j.I528-I I57.1970. tb03903.x

\section{UPCOMING SYMPOSIA}

Rheumatology

Paediatrics (RCPE/RCPCH joint symposium)

Neurology

Diabetes

Renal Medicine

Night Frights ( $T$ \& MC)

Gastroenterology

Cardiology

St Andrew's Day Symposium (Acute Medicine)

\section{Book online at http://events.rcpe.ac.uk}

\section{Live links are available to most symposia}

All symposia are held at the Royal College of Physicians of Edinburgh unless otherwise stated.

19 September

27 September

4 October

9 October

12 October

25 October

2 November

15 November

6, 7 December

Programme details at: http://events.rcpe.ac.uk or contact the Symposium Co-ordinator: Email: e.strawn@rcpe.ac.uk

\section{Webstreamed lectures}

If you are unable to attend symposia at the College in Edinburgh, selected lectures from all symposia are available to view and listen to in the Online Education Portal. http://learning.rcpe.ac.uk

There are currently more than 100 lectures covering all the medical specialties and a range of generic topics. 